De la Gazette à C.N.N., les gestes d'informer

\title{
Le visuel dans l'information
}

Du modèle à son inscription sociale

Visual aspects of news. Visual screen schedules and their impact on news.

\section{Gérard Leblanc et Jean Mouchon}

\section{(2) OpenEdition}

Journals

Édition électronique

URL : http://journals.openedition.org/edc/2680

DOI : $10.4000 /$ edc. 2680

ISSN : 2101-0366

Éditeur

Université Lille-3

Édition imprimée

Date de publication : 1 mai 1994

Pagination : 45-61

ISSN : $1270-6841$

Référence électronique

Gérard Leblanc et Jean Mouchon, « Le visuel dans l'information », Études de communication [En ligne], 15 | 1994, mis en ligne le 26 décembre 2011, consulté le 05 mai 2019. URL : http:// journals.openedition.org/edc/2680 ; DOI : 10.4000/edc.2680

Ce document a été généré automatiquement le 5 mai 2019.

(c) Tous droits réservés 


\title{
Le visuel dans l'information
}

\author{
Du modèle à son inscription sociale
}

Visual aspects of news. Visual screen schedules and their impact on news.

Gérard Leblanc et Jean Mouchon

\section{Photogramme: information et effets de grille.}

1 Quel rôle joue le visuel dans le discours global qui se présente comme celui de l'information? Toute tentative de typologie des images de l'information se heurte à plusieurs difficultés, dont la plus importante réside dans la nature de la relation du visuel à la scénarisation événementielle ${ }^{1}$, que celle-ci soit explicite ou, comme c'est le cas le plus souvent, implicite. A moins de considérer qu'un événement génère ses propres images, sa propre mise en scène (que la caméra se bornerait à enregistrer), il est impossible de les tenir pour isolées d'une scénarisation préalable, quand bien même l'événement aurait lieu en direct. Les images valorisées comme inattendues, imprévisibles, sont soumises aux critères définitoires de l'inattendu et de l'imprévisible, qui donnent lieu à scénarisation. Un événement scénarisé comme une catastrophe peut très bien n'être imprévisible que dans le discours de l'information. Quel est alors le statut des images qui accompagnent son récit, aussi «inattendues» soient-elles? N'ont-elles pas pour fonction de cultiver l'effet tragique dans des situations où la tragédie aurait pu être évitée? Ne participentelles pas à la substitution généralisée des effets aux causes? On peut être assuré en tout cas qu'elles ne sont pas autonomes, qu'elles jouent un rôle à l'intérieur d'un discours qui leur assigne une place et ne se prive pas d'ailleurs de les commenter verbalement pour mettre leur lecture sur de bonnes voies interprétatives.

2 Le visuel dans l'information vise d'abord le réel, avec lequel il est supposé entrer en contact par transparence. Il prétend n'être le résultat d'aucune scénarisation, il n'est pas mis en scène. 
Photo 1

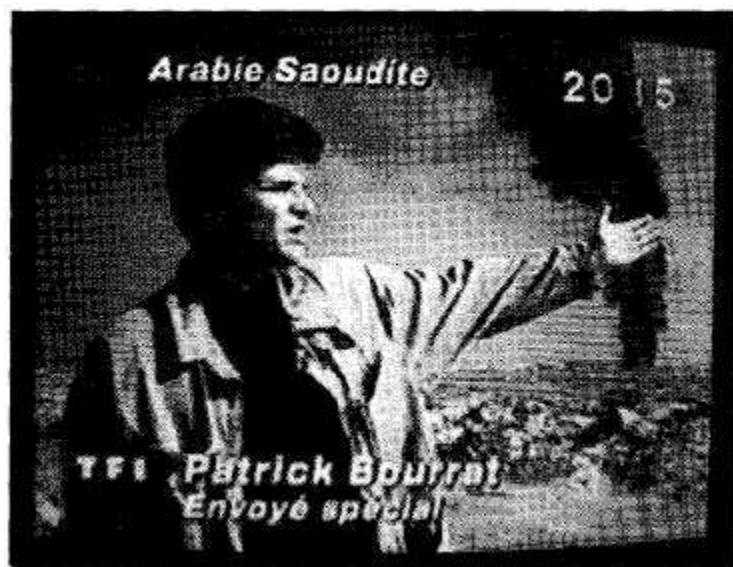

Photo 2

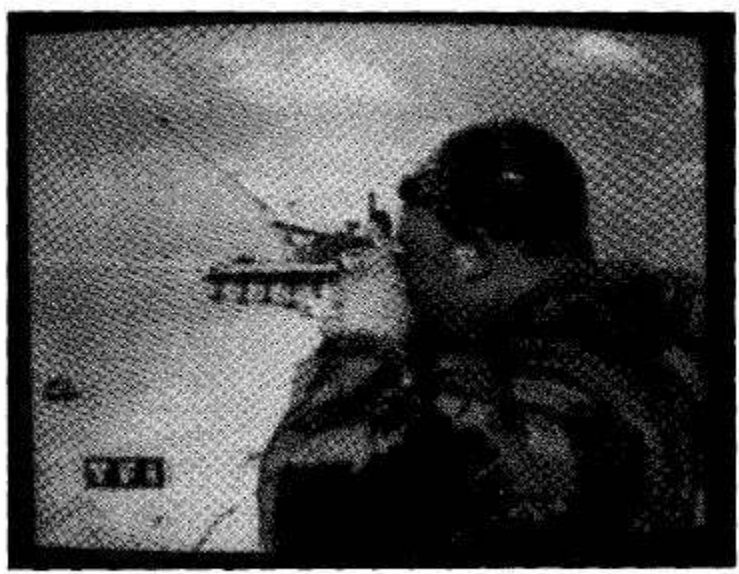


Photo 3

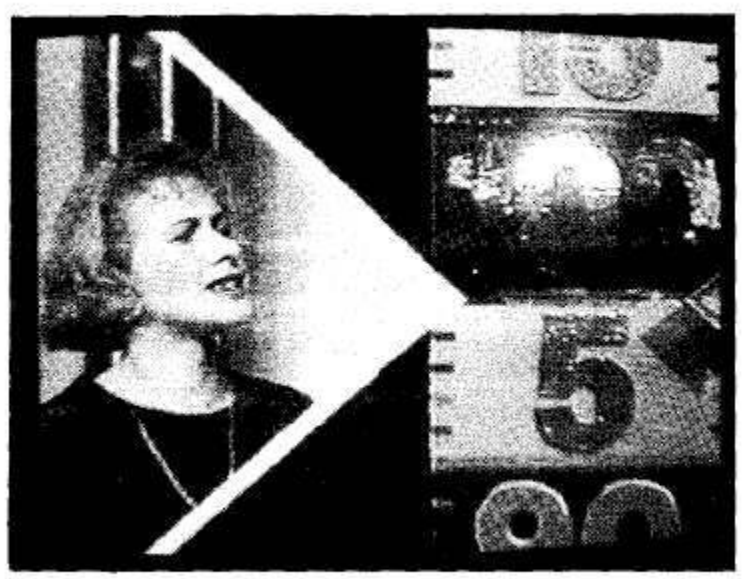

Photo 4

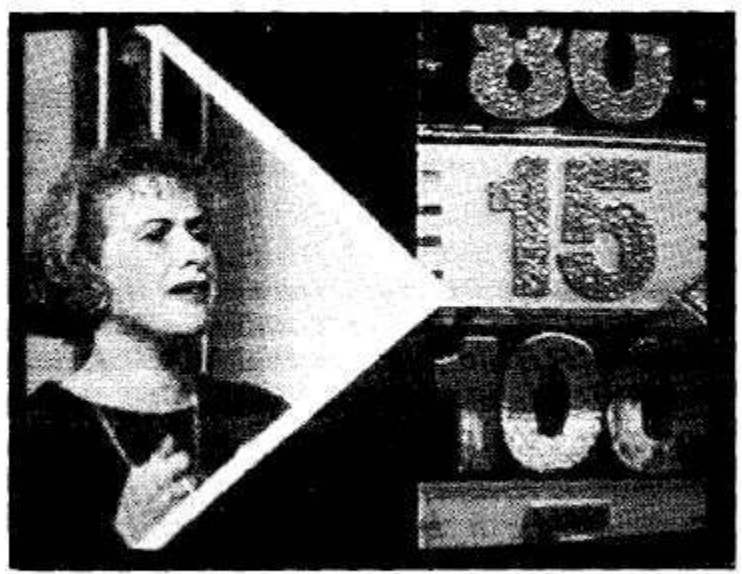


Photo 5

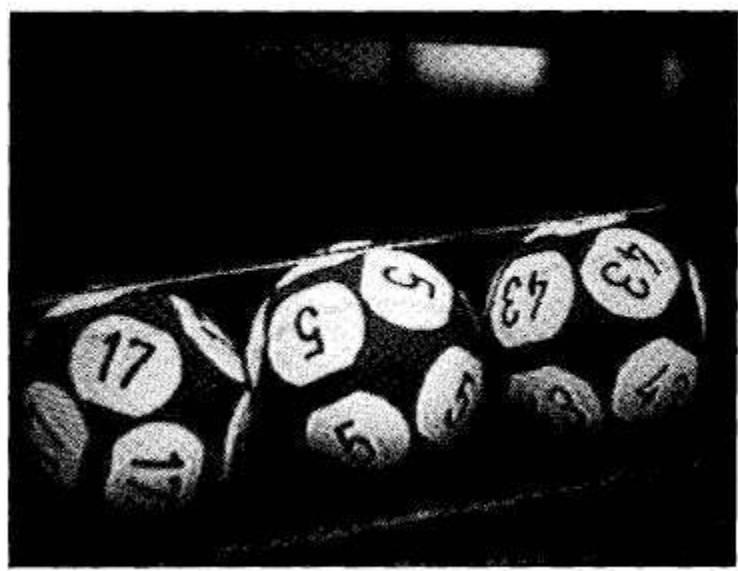

Photo 6

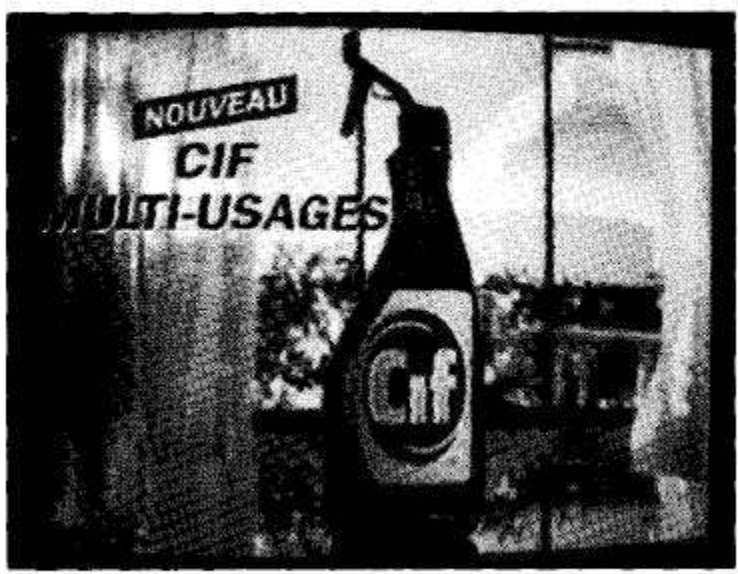


Photo 7

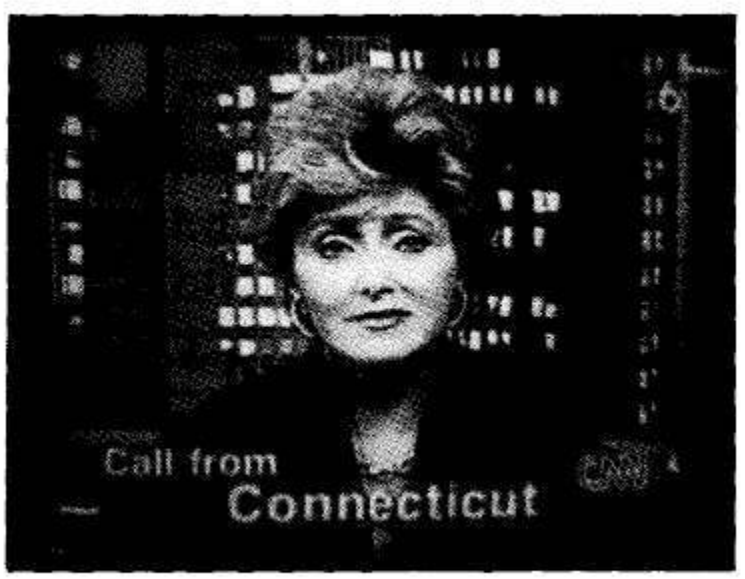

Photo 8

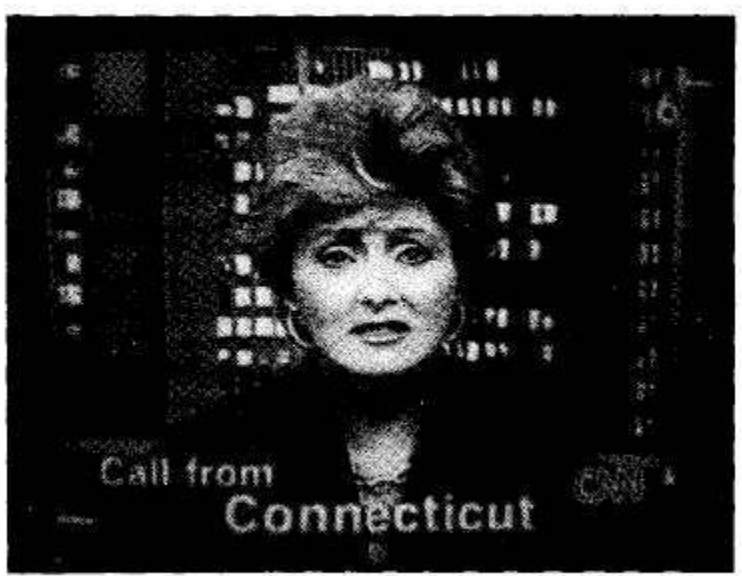

Рното 8

3 Une image n'est déclarée «spectaculaire» que parce que la réalité captée l'est déjà. Ces présupposés, si répandus dans le discours journalistique dominant, rendent le visuel - et par là le visible - aussi irrécusable que le réel, les deux termes étant substituables l'un à l'autre. Le rapport du visible au non visible - qui définit la construction de toute relation ouverte au réel - n'est jamais questionné qu'à l'occasion de l'apparition de nouvelles techniques de visualisation qui font reculer les limites de saisie du visible.

On distingue ainsi plusieurs types d'images. Il y a l'image de reportage - l'image-reine de 
l'information - où la réalité factuelle semble s'offrir sans médiations et où le direct joue un rôle prépondérant (saisie et transmission simultanée de la réalité factuelle). Il y a l'image d'archives qui permet d'insérer l'image de reportage dans une continuité interprétative de type historique (l'image de reportage devenant à son tour image d'archives). Il y a l'image d'analyse, souvent assortie de statistiques et de modèles réduits, où s'affiche la scientificité du discours du spécialiste qui la commente. Il y a l'image de synthèse qui sert à visualiser ce qui ne peut être saisi, ou n'a pu l'être au moment des faits, par les techniques de prise de vue, cinéma et vidéo. Il semble impossible de confondre ces images. De par leurs différences mêmes, elles se renforcent mutuellement, elles se complètent. Dans l'image de reportage, l'information passe par le voir; dans l'image d'archives, elle passe par la contextualisation du voir; dans l'image d'analyse, elle passe par le savoir; dans l'image de synthèse, elle passe par la re-création du voir.

parait facile à établir. Il n'est pas toujours possible de distinguer une image de reportage d'une image d'archives, car il arrive qu'une image d'archives ne soit pas désignée comme telle. L'indécidabilité temporelle et spatiale $d \sim$ certaines images insérées dans des chaînes d'images précisément datées et localisées ouvre très logiquement sur la problématique des actualités reconstituées. Les faits ont eu lieu, le direct les a couverts au mieux mais les images continuent de manquer. Or, dès que le discours de l'information télévisée se constitue en récit, la continuité narrative ne saurait s'accommoder de trop nombreuses lacunes. Pourquoi ne pas chercher alors les images manquantes dans d'autres images, déjà existantes ou spécialement créées à cette occasion? Le mode de liaison de toutes ces images, ce n'est pas la causalité et la temporalité factuelles, c'est une scénarisation dominée par une continuité narrative où seules certaines images ont un rapport direct avec la réalité factuelle envisagée.

Des images d'un même type peuvent d'ailleurs revêtir des significations bien différentes selon la fonction qui leur est assignée. Prenons l'exemple de la simulation en informatique. Qu'y a-t-il de commun entre un simulateur de vol destiné à la formation de futurs pilotes et une simulation du réel destinée à manipuler un public? Dans le premier cas, il faut que les gestes appris soient au plus près des gestes réellement pratiqués. Dans le deuxième cas, le seul impératif est de produire un effet de croyance. Seule la technique utilisée est commune aux deux situations.

L'information télévisée a déjà connu plusieurs générations d'images et plusieurs conflits de générations (entre l'image de reportage et l'image d'analyse, par exemple). Mais ces conflits n'ont jamais abouti à l'exclusion d'un type d'image par un autre. Le discours de l'information unifie les images, les rend homogènes. C'est le discours de l'information (dominé le plus souvent par un commentaire, non pas descriptif, mais narratif, scénarisé) qui assigne une place à chaque type d'image et non la relation particulière que chaque type d'image est supposé entretenir avec la réalité factuelle qui lui est extérieure.

5 L'information tend à mettre toutes les images sur le même plan, car le discours qu'elle tient sur la réalité factuelle ne prend pas sa source dans les images. Les informations contenues dans les images ne servent pas de base au discours de l'information, elles lui servent à atteindre un seuil de visibilité qui le rende convaincant. Toutes les images utilisées doivent se soumettre à l'hégémonie du visible, c'est-à-dire à l'illusion que la réalité factuelle pourrait s'offrir dans ce qu'on en perçoit à première vue. Et c'est bien parce que le conceptuel, dans le discours de l'information, se tient à l'écart du visible (ne prend pas sa source en lui) qu'il peut faire jouer à l'image un rôle tout à la fois illusoire et 
illusionniste.

L'image d'analyse, en dépit des apparences, fonctionne strictement sur le même mode. En principe, le conceptuel devrait y fonder le visible, matérialisé à l'image par des tableaux statistiques, des courbes, des schémas, des maquettes ou des modèles réduits. Mais il est exceptionnel que ce matériel didactique serve à cet usage. Le commentateur -spécialiste le montre ostensiblement («Vous voyez», lui arrive-t-il même de dire) sans l'intégrer à quelque démonstration que ce soit. Le matériel didactique est plus matière à monstration qu'à démonstration. Il sert en fait à assurer au discours du commentateur un seuil de visibilité qui le fasse apparaitre comme un discours spécialisé.

Dans tous les cas de figure, l'image est donc moins référable à une réalité factuelle donnée qu'à un discours où elle joue le rôle indispensable de preuve par le visible. Et elle est d'autant plus nécessaire au discours de l'information qu'elle lui permet d'atteindre un deuxième seuil, émotionnel celui-là. Il ne suffit pas en effet que la réalité factuelle soit identifiée au visible, et le visible à l'image. Encore faut-il que l'image regorge d'émotions qui semblent surgir à l'instant même de la réalité factuelle. Et le plus court chemin de la réalité factuelle à l'émotion, c'est le direct à suspens où rien, dans la réalité envisagée, ne paraît joué d'avance. Le reportage en direct est l'image préférée de l'information parce que, de toutes les images, c'est elle qui réalise le mieux l'alliance et l'alliage recherchés entre seuil de visibilité et seuil émotionnel.

6 Tout pris et compris qu'il soit dans un discours déjà scénarisé, le visuel dans l'information joue un rôle qui n'est pas réductible à ce discours, même s'il en est étroitement dépendant. Le visuel signifie aussi par lui-même. Sans viser ici à l'exhaustivité, nous allons maintenant étudier deux cas de figure où le visuel fait sens, à l'ombre du discours verbal qui le surplombe sans l'épuiser. Si le visuel parle " par lui-même », c'est d'abord en ce qu'il assigne une place et un positionnement - intellectuel, affectif - particuliers au (télé)spectateur. Il induit un rapport au réel à travers des images qui ne se contentent pas d'illustrer un discours.

\section{Le geste auguste du grand reporter. (photo 1)}

7 « Où que je sois, je suis là où il faut au moment où il faut. Absorbé par l'image, je suis dans cette image où l'on me presse d'entrer ».

Ces quelques mots pourraient donner sens à cette image, itérative s'il en est à la télévision. Par la médiation du grand reporter - mon délégué, mon envoyé spécial - je suis présent aux convulsions du monde. Son geste auguste - qui désigne, circonscrit, délimite une portion de l'espace - est la preuve visuelle du réel auquel sa parole se réfère. Son geste me dit: «vous voyez ", et ce voir implique croyance et confiance en sa parole. Elle est vraie. Je vois en cet instant - c'est ce qu'il me dit - que «tout est calme» au Koweït. La portion d'espace désignée par le geste s'enfle aux dimensions d'un pays, voire du golfe arabe tout entier. Il semble alors que la parole excède largement la preuve visuelle proposée par le geste. De fait, l'image matérielle fait place à une représentation beaucoup plus générale à laquelle elle a servi de rampe de lancement. «Tout est calme» ne signifie pas seulement le calme dans cette image, les dernières fumées d'une bataille gagnée ou perdue, il signifie la perte de la guerre par l'Irak et la victoire des forces coalisées. La valeur symbolique se substitue à la valeur descriptive.

Il est fréquent que la valeur descriptive d'une image soit utilisée à des fins de généralisation (sans que cette image ne l'autorise en rien, elle fournit seulement un 
prétexte à une généralisation venue d'ailleurs) ou même, parfois, à des fins d'intervention politique. Voici le ministre Bernard Kouchner, en 1992, dans les habits du grand reporter. Il se trouve dans un hôpital somalien (de fortune, comme on dit), au milieu d'enfants agonisants. «Que faire pour la Somalie? interroge-t-il en regardant le téléspectateur droit dans les yeux (pendant ce temps, ajoute-t-il en substance, il y a les Jeux Olympiques de Barcelone et les occidentaux ne pensent qu'à ça). Tout en parlant, le ministre tient la main d'un enfant somalien agonisant, et la main retombe. L'émotion générée par ces images de reportage devient politique dans la mesure où le ministre s'est substitué au reporter dans une situation qui s'apparente à celle du grand reportage. Sa fonction ne lui permet pas de s'en tenir à la valeur descriptive de l'image, alors que la fonction du grand reporter voudrait qu'il s'y tienne.

Il arrive parfois que des grands reporters se contentent de restituer ce qu'ils ont vu et entendu (c'est la déontologie du métier). Volontiers redondant, Hervé Brusini s'est souvent illustré, ces dernières années, dans cette posture. "Vous avez un exemple derrière moi, en direct ", nous dit-il un jour. La parole du reporter est ancrée dans le réel dont témoigne l'image (les deux se confondent), mieux, son corps est immergé dans le réel dont il parle. Et d'un geste de la main (toujours ce geste, ample et décisoire, en forme de constitution de preuve visuelle), il désigne « derrière lui» l'empilement de cercueils dont il vient de parler.

Contrairement à ce qui est trop fréquemment dit et écrit, les images de grand reportage ne donnent pas le monde en spectacle (elles ne le traduisent pas en images), elles le donnent à ressentir dans son immédiateté phénoménale, au prix de sa réduction au visible et, plus largement, à ses apparences sensibles (et le visible, nous le savons trop bien, peut faire l'objet de toutes les manipulations possibles). La confusion vient du fait que les grands reporters cèdent souvent au désir de scénariser les risques qu'ils prennent dans l'exercice de leur métier. Voici qu'un reporter nous annonce l'interview de la fiancée d'un «chef de bande» dans un immeuble aux trois quarts détruit de Sarajevo. Nous n'assistons pas immédiatement à l'interview. Le reporter entend d'abord nous montrer le parcours du combattant qu'il doit accomplir pour accéder à cet immeuble particulièrement exposé. Pour arriver à l'appartement où l'attend la future interviewée, le reporter doit s'exposer lui-même, nous le voyons courir à plusieurs reprises d'un point à un autre, à découvert, il pourrait être tiré à chaque instant comme un lapin. Mais cette mise en scène d'un risque effectif n'empêche pas le téléspectateur de considérer que la situation est réelle (non fictive) et de s'impliquer dans l'action avec son «envoyé spécial ». Si le téléspectateur est présent au monde par la médiation du reporter, c'est bien le monde réel, tel qu'il lui est présenté, qui mobilise et motive ses réactions intellectuelles et affectives, et non la transformation du monde réel en spectacle.

\section{Dedans/dehors: la question du point de vue. (photo 2)}

8 Qu'elles soient ou non médiatisées par la figure du reporter, les images de reportage se différencient selon qu'elles prétendent nous introduire à l'intérieur des choses, dans leur mouvement et surgissement, ou selon qu'elles nous laissent à l'extérieur, dans la position du témoin. C'est le problème de la constitution du point de vue, qui n'est jamais énoncé comme tel dans le discours journalistique et reste toujours implicite. Voici par exemple un tir effectué au cours de la guerre du Golfe. Par l'angle de prise de vue choisi, par la proximité ménagée par le plan rapproché, je suis placé dans le mouvement de ce tir, je le 
partage avec ceux qui en ont la charge. Je suis, politiquement et militairement, de leur côté. Un tout autre positionnement du téléspectateur aurait été obtenu en enregistrant le même tir en plan général, sous un angle qui ne situe pas le téléspectateur dans le prolongement de l'arme (l'érigeant en utilisateur potentiel), et aussi, et surtout, en montrant la guerre des deux côtés et non d'un seul. Les effets politiques induits par ce positionnement du téléspectateur sont renforcés par la puissance énergétique qui lui est conférée. «J'ai l'oeil surarmé», pourrait dire le téléspectateur, «la puissance de feu de mon regard me rend invincible ». Cette illusion de toute-puissance, par la satisfaction narcissique qu'elle lui procure, invite le téléspectateur à s'identifier à la supériorité technologique des forces coalisées, dans l'oubli des causes réelles d'une guerre qui doit être absolument gagnée: son regard, aussi, est en jeu.

Le mode d'implication du téléspectateur dans une action est bien différent selon qu'il est « dedans» ou "dehors ». Grâce à une caméra placée à l'intérieur de la voiture, il avale avec Alain Prost un circuit de formule 1, ses lignes droites et ses virages. Il partage ses sensations de pilote. Autre moment de la course. Suite à un accrochage, une voiture sort de la route, le moteur prend feu, le bolide fait plusieurs tonneaux, perd ses roues, etc... Le téléspectateur n'est plus dans la voiture, il ne regarde plus et ne ressent plus le monde du point de vue du pilote, il assiste à une action sans y participer directement. L'extériorité n'annule pas l'émotion, elle lui confère une dimension objective où les deux mondes (celui du téléspectateur et celui de la course) interagissent sans jamais se confondre. La question est ici de savoir dans quel état le pilote va se sortir de l'accident dont il vient d'être victime (direct à suspens). On observera que la forme moderne du reportage sportif (qui sert de modèle à d'autres formes de reportage) s'efforce de combiner les deux points de vue, l'intégration du premier - «l'être avec» - permettant d'augmenter l'intensité des sensations et des émotions générées par le second.

L'impact d'une image n'est donc pas lié au caractère inédit ou répétitif de la scénarisation événementielle à laquelle elle se rattache nécessairement (quoi de plus répétitif, de moins inattendu, d'ailleurs, que la plupart des événements présentés comme imprévisibles, dès lors qu'on les rapporte aux processus dont ils résultent). L'impact d'une image de reportage dépend de sa capacité à nous faire participer - en tant que téléspectateur - à un événement, quel qu'il soit - imprévisible ou programmé, itératif ou inédit, déclaré important ou mineur, avec autant d'intensité que possible. La réussite d'une image de reportage est de s'abolir en tant qu'image pour faire place aux choses mêmes.

Si Sarajevo est bien réel - et la télévision me le fait vivre comme tel - mon réel n'est pas celui de Sarajevo. Mon envoyé spécial, le grand reporter, convertit la représentation en présence, mais l'événement qui m'avait tant ému - à chaud, sur le coup -, et dont j'étais devenu un des acteurs virtuels, ne survit guère à sa disparition. La vie continue. Ainsi le veulent les grilles de programmes. La guerre est quotidienne à la télévision, mais mon quotidien n'est pas celui de la guerre, c'est celui de la paix occidentale, ponctué par des jeux et des émissions de divertissement. C'est d'ailleurs dans les jeux et les émissions de divertissement que la télévision me renvoie ma propre image, alors qu'elle n'apparaît jamais, même incrustée, sur le théâtre des opérations guerrières. Il est néanmoins important de remarquer que la guerre existe potentiellement dans bien des émissions qui s'apparentent à des émissions de divertissement. Ainsi en va-t-il, par exemple, des compétitions sportives où il s'agit de vaincre un adversaire qui devient rapidement un ennemi, voire même - métaphoriquement - de le «tuer» (on se référe ici à la célébre théorie selon laquelle on ne saurait devenir un grand joueur de tennis sans avoir la 
mentalité d'un «tueur»).

Il semble alors légitime de parler d'effets de grille, à propos d'images qui jouent un rôle au-delà du champ de scénarisation qui leur est propre pour influer sur le comportement global du téléspectateur, y compris sur sa relation au monde construit par le discours de l'information. On peut dessiner à partir de ces images de véritables figures emblématiques, dont toute la force - qui naît, en partie, de leur répétition - consiste à concentrer un rapport au monde porté à son maximum d'intensité. En voici trois exemples, centrés autour du ludique. Il y en a beaucoup d'autres.

\section{Mon semblable, mon frère (ma soeur): le comble de l'émotion. (photos 3 et 4 )}

Voici le visage anxieux de celle qui est supposée me ressembler (ainsi en ont décidé les sondages). La roue de la fortune est en train de tourner et, du 5 au 95, son visage passe par toutes les émotions et, instantanément, d'une émotion à l'autre, de la plus folle espérance à la plus sombre angoisse. Comme si sa vie était en jeu, comme si elle ne tenait plus qu'à ce fil ténu qui la relie à cette roue qui tourne, distribuant, selon le chiffre auquel elle s'arrête, le bonheur ou le désespoir. Aucun autre genre d'émission (hormis, sur un autre mode, les «reality shows») ne met à ce point en scène l'émotion du candidat téléspectateur. Combien de candidats au souffle raccourci, au regard défaillant, au bord de l'évanouissement? Nul autre événement au monde ne saurait générer une telle charge émotionnelle.

Présent à la télévision, la télévision me rend présent au monde. Il y a là un phénomène de double présence simultanée: du téléspectateur à la télévision - réception -, de la télévision à l'événement - émission. La distance s'abolit dans l'immersion, dans la suppression des médiations (la principale fonction du médiateur - grand reporter ou présentateur - étant de s'abolir en tant que tel pour faire entrer le téléspectateur dans l'événement, comme un de ses acteurs virtuels). Mais il y a monde et monde. Si celui de l'actualité est aussi quotidien que celui des jeux, s'il peut même revêtir l'aspect d'un jeu (comme au moment de la guerre du Golfe, où le théâtre des opérations était transformé en réalité virtuelle, où le regard tout puissant du téléspectateur était doté d'armes sophistiquées dont il pouvait user avec une extrême précision - les enfants disposant quant à eux de modèles réduits), le téléspectateur n'y engage ni son image ni son mode de vie. La guerre fait partie de la panoplie des jeux disponibles - certes plus dangereux qu'un autre - alors que le monde des jeux présente au téléspectateur l'image globale du mode de vie - pacifié, heureux: consommateur - dont les jeux font partie.

\section{La part du hasard: figure de l'imprévisible. (photo 5)}

11 Nous étions dedans, avec les candidats, ces autres nous-mêmes. Nous sommes maintenant dehors, dans le suspens de l'événement annoncé: les résultats du tirage du Loto. Aussi légères que des bulles, les petites boules s'agitent dans le globe qui les contient, elles vont sortir dans un certain ordre, composant une à une les chiffres du numéro gagnant. Nous faisons peut-être partie des millions de français qui jouent au loto et sommes alors directement intéressés aux résultats. Mais le véritable impact de cette image est ailleurs: dans cette alliance, toujours reconduite, de la programmation et de l'imprévisible. Comme si la programmation ne pouvait échapper au hasard, mieux, comme si le hasard 
en était le lieu et l'enjeu.

Effet de grille là encore, qui opère par contagion et déplacement. La scénarisation de l'information n'oppose-t-elle pas en permanence la programmation étatique, gouvernementale, institutionnelle - et ce qui lui échappe? La tendance dominante n'estelle pas de présenter tout phénomène qui déroge à l'ordre présupposé normal du monde naturel ou social- sous le signe du hasard et de l'imprévisible? Et si, par hypothèse, le devenir du monde échappait toujours davantage aux pouvoirs qui tentent de le maitriser, ne faudrait-il pas aussi qu'augmente toujours davantage, dans la représentation du monde, la part du hasard et de l'imprévisible? Il faudrait alors que la part des jeux de hasard, sous des formes variées et multiples, augmente toujours davantage dans les grilles de programmes.

Cette figure de l'imprévisible se substitue à la part effective de hasard qui existe dans le devenir du monde. Elle favorise la confusion entre les événements qu'il est possible, sous certaines conditions, de maitriser, et ceux qui sont réellement imprévisibles. Elle nous habitue à l'idée que la programmation rencontre partout le hasard et qu'aucun résultat escompté n'est assuré d'être atteint.

\section{Le geste publicitaire: figure de la transparence. (photo 6)}

12 Une main féminine glisse sur une vitre. Comme l'atteste le sourire angélique de la femme, le geste est facile, merveilleusement efficace. La vitre devient si transparente qu'on finit par se demander si elle existe encore. C'est que le geste est relayé par le bon produit (CIF, en l'occurrence) et cela suffit pour le rendre magique.

On peut douter de l'efficacité commerciale d'une telle représentation. La magie publicitaire s'use à force de lessives qui, contrairement aux promesses, ne lavent guère plus blanc que les autres. Mais cette image participe d'une représentation beaucoup plus générale où le monde - sans résistance, sans contradictions, sans exigence d'effort s'accorderait au désir. La construction d'un monde imaginaire où le désir règne en seul maitre, qui est le fond de l'entreprise publicitaire, dissuade le spectateur d'affronter le monde réel, où le désir rencontre continûment des obstacles. Le désir de transparence ne peut se réaliser que dans l'univers de la consommation. On peut du moins s'en remettre à l'illusion qu'il suffit du bon produit ? La fin s'est trouvé un moyen facilement identifiable et à la portée de tous.

\section{Un mode d'adresse privilégié: le regard de veille. (photo 7)}

13 On ne saurait suspendre ces analyses de cas sans explorer un des cas de figure où la télévision s'adresse à nous, directement et sans médiations. Ici, la suppression des médiations n'a pas pour but de nous donner l'illusion de participer à la réalité factuelle et il ne s'agit pas non plus de nous renvoyer notre propre image, prise et comprise de toutes parts dans le circuit de la consommation. Il s'agit de nous prendre en charge dans les situations d'angoisse et de détresse que nous pourrions être amenés à vivre - la télévision les aurait-elle provoquées elle-même. La télévision veille sur nous en toutes circonstances - dans le malheur comme dans le bonheur -, elle ne nous abandonnera jamais. 
Regardons. Ce regard dirigé vers nous (photo 8) est un regard empli de compassion, un regard qui écoute de toutes ses oreilles l'expression orale de notre malaise. Nous sommes aux Etats-Unis, sur la chaîne CNN, pendant la guerre du Golfe. Une voix féminine raconte qu'elle a tendance à confondre une sirène d'ambulance avec une alerte à la bombe. Comme si les rôles étaient inversés, comme si l'Irak se trouvait en position de pilonner les Etats-Unis. A n'en pas douter, cette femme va très mal. Faut-il parler de confusion mentale? Le visage compatissant ne se hasarde pas à émettre un diagnostic. II s'agit moins de soigner une personne malade que de traiter des pouvoirs de la télévision. Le visage à l'écoute se met à parler à son tour, d'une voix douce, insinuante, rassurante. Il est question de la merveilleuse capacité du direct à nous faire vivre les événements et des dangers qui en découlent. Quand un direct nous amène à vivre un événement dramatique, comment le drame que nous avons vécu ne rejaillirait-il pas sur notre propre vie? C'est bien là que se trouve la difficulté: vivre intensément un événement dramatique et passer, aussitôt après, à autre chose. Les grilles de programmes favorisent la réintégration du téléspectateur dans la vie ordinaire: d'autres émotions, tout aussi intenses, lui sont proposées, mais liées cette fois à son quotidien (qu'il s'agisse d'un jeu ou d'un «reality show»). Mais si la télévision a le pouvoir de faire vivre au téléspectateur toutes sortes d'événements de l'intérieur, y compris ceux qui paraissent à mille lieues de sa vie ordinaire, le téléspectateur doit y mettre également du sien. Il doit «faire attention» (c'est du moins le conseil qui lui est prodigué): attention à ce qu'une vie ne déteigne pas, ne déborde pas sur l'autre. On pourrait ajouter, sans forcer le sens du discours, que le téléspectateur doit concevoir sa vie à l'image d'une grille de programmes.

\section{Quelques pistes de recherche.}

14 Limités en nombre, ces quelques exemples n'ont pas été choisis de façon arbitraire. Leur analyse permet de mieux comprendre les enjeux du visuel dans l'information.

Le visuel dans l'information a pour première fonction d'accentuer l'écart entre le visible et la réalité factuelle, par assimilation de l'un à l'autre. Il est rarissime qu'un discours de reportage mette en avant les limites de visibilité d'un événement donné. La réalité est ouverte, sans doute, elle est en suspens, mais c'est dans le visible que l'événement se déroule et, éventuellement, se dénoue, et non dans la construction d'un rapport au réel fondé sur l'interaction visible-non visible ${ }^{2}$.

Le visuel dans l'information intervient dans la construction d'un rapport au réel marqué par la substitution du réel à l'image. Par le mode d'implication qui lui est propre, il ne constitue pas celui qui regarde en spectateur mais en acteur des événements avec lesquels il est supposé interagir. Le visuel ne focalise pas l'attention sur l'image, bien au contraire, il tend à faire oublier son existence ${ }^{3} . I l$ permet alors au discours verbal de procéder à des généralisations que lui interdirait l'image, si elle était prise en considération dans sa valeur descriptive.

Le comportement du téléspectateur est modelé par des effets de grille, qui influent aussi bien sur sa conception du monde que sur ses réactions émotionnelles. Le monde de l'actualité, aussi dramatisé soit-il, tend à être vécu sur un mode ludique. 


\section{BIBLIOGRAPHIE}

Ce texte est issu d'une série d'articles publiés, sous forme de chronique, dans la revue l'Image Vidéo (Editions Fréquences):

L'Image Vidéo, (décembre 1989), «L'actualité tragique», Editions Fréquences, n 1

L'Image Vidéo, (février 1990), «Les scénarios du réel », Editions Fréquences, nº 2.

L'Image Vidéo, (avril 1990), «La mise en charnier », Editions Fréquences, nº 3.

L'Image Vidéo, (juin 1990), «Les images de l'information», Editions Fréquences, $n^{\circ} 4$.

L'Image Vidéo, (septembre 1990), «Vivre avec la télévision», Editions Fréquences, nº 5.

L'Image Vidéo, (novembre 1990), «Vacances en Irak», Editions Fréquences, nº 6.

L'Image Vidéo, (janvier 1991), «Les images virtuelles de la poésie », Editions Fréquences, nº 7.

L'Image Vidéo, (mars 1991), «Sur la terre comme au ciel», Editions Fréquences, $n^{\circ} 8$.

L'Image Vidéo, (mai 1991), «La vitesse et la lumière», Editions Fréquences, $n^{\circ} 9$.

\section{NOTES}

1. La notion de scénarisation du factuel est liée à la conception dominante de l'information, fondée sur l'information-infraction. Dans la dissociation qu'ils opèrent entre les faits et les processus dont ils résultent (dissociation qui est à la base de l'information-infraction), les scénaristes du factuel tendent à calquer la réalité sur un schéma narratif qui présuppose la perturbation d'un ordre initial, naturel, normal, stable. La scénarisation se concentre sur la perturbation (le désordre), enregistre le conflit qui en résulte (l'affrontement entre les forces du désordre et les forces de l'ordre), tend à montrer, et souvent contre toute évidence, le retour à un état d'équilibre (maîtrise de la perturbation, sinon dans ses causes, du moins dans ses effets). Autrement dit, cette forme de scénarisation du factuel postule que le schéma narratif qu'elle intègre se trouve déjà dans le réel. Aussi borne-t-elle son appréhension du factuel à des constructions fictionnelles déjà connues sans jamais questionner leur capacité d'élucidation. Elle cherche à retrouver dans le réel les scénarios auxquels la conception dominante de la fiction nous a dépuis longtemps habitués.

2. Depuis quelques mois, les journaux télévisés reviennent plus volontiers sur les processus qui ont abouti à des catastrophes présentées habituellement comme «naturelles» et saisies essentiellement dans leurs effets. Aussi peu affirmée que soit encore cette tendance, elle est encourageante et pourrait mettre en cause, à plus ou moins long terme l'économie générale de l'information.

3. Cette assimilation du voir au vivre évoque bien entendu le monde des images et des réalités virtuelles. Si l'information n'utilise aujourd'hui ces techniques que de façon secondaire, si l'interactivité proposée n'est pas une interactivité effective, il s'agit bien de donner à vivre, sur un mode fantasmatique, un monde simulé comme un monde réel, dans l'oubli des règles qui ont présidé à sa constitution. 


\section{RÉSUMÉS}

Les auteurs se proposent d'analyser l'apport propre au visuel dans l'information télévisuelle. Images de reportage, images d'archives, images d'analyse ou de synthèse semblent couvrir au mieux le réel; néanmoins elle continuent de manquer et c'est le discours d'information, par sa scénarisation, qui fait lien et continuité. Il assigne une place au visuel et le commente.

Les auteurs posent que le visuel n'est pourtant pas réductible au discours. Issues souvent d'autres types de programme que de l'information télévisée, plusieurs figures, analysées dans cet article, construisent, par leur effet de grille, le rapport du spectateur à ce qui est montré. Elles concentrent un rapport au monde porté à son maximum d'intensité et leur force vient de leur répétition. La première, la Roue de la Fortune en fournit un exemple, offre la charge émotionnelle d'image de personnes aux modes de vie proches du téléspectateur. La deuxième, avec le Loto, montre ce qui échappe à la programmation sous la figure du hasard, de l'imprévisible. La troisième, avec la communication publicitaire, propose un monde où le réel est sans résistance, pour peu qu'on dispose du bon produit. Enfin le visuel est parfois une adresse directe au spectateur, celle d'un visage empreint de compassion, figure qui permet de passer de l'intensité dramatique de l'événement aux émotions tout aussi intenses de la vie ordinaire.

Ce visuel dont le mode de lecture est installé tout au long de la grille de programme, joue encore au moment où se donne à entendre le discours de l'information.

The authors want to demonstrate the visual sequences cannot be reduced to the meaning of speech. Reports in pictures, pictures from archives, analytical or synthetic pictures are supposed to be the best way to cover the real. However they are continually missing it and the informative speech provides the links and continuity. It seUles the picture a place of their own and produces a commentary.

According to the authors the visible cannot be submitted to speech. they analyse several kinds of pictures appearing more frequently in other type of programmes which establish a relation of the watcher to what he is shown through the impact of their repeated patterns.

The first type of those weeckly or daily programmes La Roue de la Fortune offers an example by introducing ordinary people whose way of life is quite similars to the watchers'one, which produces an emotional effect. The second type, the Bingo, shows as risky and unpredictable all that escares a scheduled series. As for the third type, the commercial, it gives the idea of a uni verse deprived of any resistance as long as you can use the most convenient product. Then, at last, the screen picture addresses the TV watcher straight through the feeling of compassion expressed by the announcer's face, which allows an immediate passage from intensely dramatic facts to the equaly intense emotions of everyday life.

These visual patterns which are recurrent in TV programmes keep their impact in the watchers' mind while the announcer is developping his speech about the news. 
INDEX

Keywords : information representations, image analysis, television, journalism, semiology Mots-clés : représentation de l'information, analyse de l'image, télévision, journalisme, sémiologie

\section{AUTEURS}

GÉRARD LEBLANC

Gérard Leblanc, Université Paris 3

JEAN MOUCHON

Jean Mouchon, ENS Fontenay/Saint-Cloud 